\title{
Le portail occidental de la cathédrale de Lausanne : tradition et modernité d'un grand chantier gothique
}

\section{Dave Lüthi}

\section{(2) OpenEdition}

\section{Journals}

Édition électronique

URL : http://journals.openedition.org/edl/1449

DOI : $10.4000 /$ edl. 1449

ISSN : 2296-5084

Éditeur

Université de Lausanne

\section{Édition imprimée}

Date de publication : 1 décembre 2018

Pagination : 291-310

ISBN : 978-2-940331-69-7

ISSN : 0014-2026

\section{Référence électronique}

Dave Lüthi, « Le portail occidental de la cathédrale de Lausanne : tradition et modernité d'un grand chantier gothique », Études de lettres [En ligne], 3-4 | 2018, mis en ligne le 15 décembre 2020, consulté le 21 décembre 2020. URL : http://journals.openedition.org/edl/1449; DOI : https://doi.org/10.4000/ edl. 1449 


\section{LE PORTAIL OCCIDENTAL DE LA CATHÉDRALE DE LAUSANNE: TRADITION ET MODERNITÉ D'UN GRAND CHANTIER GOTHIQUE}

Les modifications apportées au massif occidental de la cathédrale de Lausanne sous l'épiscopat d'Aymon de Montfalcon bouleversent profondément l'aspect et le fonctionnement liturgique de l'édifice. En dépit de leur nouveauté formelle, les parties ajoutées par l'évêque se placent dans une certaine forme de continuité dont on peut interroger le sens. Visiblement intéressé par le recours à l'histoire, sans doute dans la perspective d'asseoir son pouvoir, le prélat fait œuvre d'archéologue avant l'heure. Mais ce n'est peut-être pas son seul but: imprimer sa marque personnelle dans l'édifice dissimule sans doute des intentions restées sans lendemain, comme celle de fonder une collégiale privée.

La construction d'un portail à l'ouest de la cathédrale de Lausanne est un événement tant pour l'histoire du monument que pour celle de l'architecture régionale. L'évêque Aymon de Montfalcon apporte des modifications essentielles à l'église en lui donnant une forme sans doute plus conforme à ce que l'on attendait alors du siège du pouvoir épiscopal. L'étude minutieuse des sources et des artisans du portail menée par Karina Queijo et par Marcel Grandjean dans ce volume amène des éléments importants pour comprendre ce chantier et le situer dans son contexte architectural tant régional qu'international, mais toutes les questions concernant l'originalité de la forme du portail ne sont pas résolues pour autant. En recentrant le discours sur le rapport du portail à la cathédrale, de nouvelles pistes de réflexions émergent, qui devront être confrontées aux hypothèses antérieures pour en valider la pertinence. 


\section{Le contexte monumental}

Rappelons ici succinctement quelques éléments de la topographie du monument tout à fait étonnants, qu'Aymon de Montfalcon a fait disparaître lors des travaux qu'il commande en 1515 et qui expliquent en grande partie les dispositions réalisées par les architectes Magyn et Contoz. Depuis la fin du grand chantier des XII ${ }^{e}$-XIII ${ }^{\mathrm{e}}$ siècles, vers $1230^{1}$, la cathédrale était percée à l'extrémité occidentale de sa nef par un passage routier reliant les parties sud et nord de la Cité et qui séparait la partie consacrée du massif occidental, sans fonction liturgique dans sa partie basse ${ }^{2}$. Au niveau du triforium, une profonde tribune surmontait le passage et créait un lien visuel vers les chapelles hautes du massif, étagées en tribunes selon un principe qui n'est pas sans rappeler les Westwerk carolingiens et romans. Ce dispositif pourrait être avant tout le fait des besoins visuels spécifiques impliqués par l'existence du passage large d'une travée et demie, fermé du côté du sanctuaire par un mur que perçait au moins un portail central (la véritable porte occidentale de l'église, en fait) ${ }^{3}$. Les fidèles arrivant de l'ouest, par les escaliers du Marché notamment ${ }^{4}$, se trouvaient donc confrontés à une façade certes inachevée, mais néanmoins monumentale - Alain Villes l'a récemment décrite comme un arc de triomphe dédié à la Vierge ${ }^{5}$ - percée en son centre par une haute arcade encadrée par les deux contreforts latéraux des tours. Cette arcade surhaussée (deux niveaux de colonnettes la

I. Pour la chronologie, nous nous fondons sur l'étude fondamentale de M. Grandjean, «La cathédrale actuelle».

2. M. Grandjean, "Le magnum portale de la cathédrale de Lausanne et le passage routier de la "grande travée" ".

3. La terminologie est ici essentielle: selon J.-M. Pérouse de Montclos, un portail est une composition monumentale comportant des portes, qui sont elles-mêmes des ouvertures fermées par des vantaux permettant un passage. Un porche est un espace ouvert devançant une porte ou un portail. Le type d'entrées que l'on trouve à Lausanne est délicat à décrire puisque les baies qui la composent ne sont ni portes, ni portail: nous avons donc opté pour le vocable neutre d'arcade, comme Marcel Grandjean l'avait fait avant nous (J.-M. Pérouse de Montclos, Architecture).

4. Le mur de ville étant placé très près de la façade, le portail occidental n'était sans doute pas l'entrée la plus usitée: la création d'un porche au sud vers 1230 en remplacement d'une porte plus modeste semble montrer que l'accès depuis la Cité-Dessous était le plus emprunté.

5. A. Villes, «La cathédrale actuelle», p. 82 sq. 
portent) donne accès à un porche voûté portant la plus haute des tribunes du massif (fig. 1). Ce porche présente un plan unique en son genre: la travée centrale, carrée, est accostée de part et d'autre par deux absides dont la partie supérieure présente des colonnettes similaires à celles de l'arcade d'entrée. Elles supportent d'étranges demi-arcs diaphragme qui soutiennent des voûtains presque plats et qui donnent l'illusion d'une couverture similaire à celle du chœur, par exemple. Une arcade assez sobre, en arc brisé, surmontée d'une claustra de pierre devant laquelle est assise la Vierge en majesté - aujourd'hui fragmentaire - dans une niche à baldaquin, donne accès à une travée carrée ${ }^{6}$. Celle-ci est délimitée par les ébrasements profonds de quatre piles flanquées de colonnettes supportant les voussures des arcs portant la voûte d'ogive, également sobre, de cet espace de transition, ouvert à l'origine sur ses quatre faces. Ensuite, il fallait traverser le passage routier aujourd'hui disparu, voûté sans doute lui aussi, pour arriver par une porte disparue, mais attestée par les sources, dans la nef elle-même. On ignore quels étaient les systèmes de fermeture de ces différents espaces; mais sans doute, au vu de la taille des baies, toutes celles du massif n'étaient que des arcades ouvertes, sans huisseries particulières, mais peut-être fermées par des grilles ${ }^{7}$; seule la porte percée entre le passage routier et la nef devait être fermée par des vantaux de bois qui portaient peut-être l'une des deux appliques de bronze qui se voient aujourd'hui encore sur les portes néogothiques du portail rénové d'Aymon de Montfalcon ${ }^{8}$.

Lorsque le portail occidental est construit au $\mathrm{XVI}^{\mathrm{e}}$ siècle et le passage routier bouché par deux murs latéraux, la géographie de la Cité s'en trouve profondément modifiée. Une maison sise près de la tour nord doit être détruite pour permettre le passage des personnes et des convois; dorénavant, on doit donc contourner la cathédrale, ce qui donne encore plus de visibilité au portail à venir, puisque l'on passe presque forcément

6. Voir la figure 1 de l'article de K. Berclaz dans ce volume.

7. Elles semblent toutefois rares dans la région avant la fin du Moyen Âge (M. Grandjean, "Euvres majeures de la ferronnerie en Suisse romande à la fin de l'époque gothique», p. 203). Un fragment de grille datée des XIVe-XVe siècles est conservé au lapidaire de la cathédrale (Cathédrale de Lausanne. $700^{e}$ anniversaire de la consécration solennelle, p. 200, cat. 183).

8. S. Utz, "Dans la gueule du lion», p. 39. Lapplique la plus ancienne date sans doute du début du XIII ${ }^{\mathrm{e}}$ siècle, la seconde est une copie libre du XVII ${ }^{\mathrm{e}}$ siècle. 
Fig. 1 - Revers du portail Montfalcon avec l'arcade du XIII siècle, premier «portail» de la cathédrale de Lausanne. Photographie Jeremy Bierer, 2012. 
devant lui ${ }^{9}$; son rôle ornemental est donc majeur ${ }^{10}$. Mais à l'intérieur aussi, cette création a des conséquences importantes: toute la succession des espaces d'entrée - une surface considérable: porche à absides latérales, vestibule entre les deux tours, puis ancien passage routier, soit près de 400 mètres carrés - se trouve intégrée à l'église, de même que les deux locaux situés sous les tours; celui du nord deviendra d'ailleurs la chapelle de l'évêque Aymon de Montfalcon. L'omniprésence des signes attestant de l'intervention de l'évêque (armoiries, devise) ne manque pas de frapper; ces «signatures" sont là pour rappeler que le prélat finance les travaux, mais ils peuvent aussi signifier l'intérêt qu'il trouve visiblement à modifier, compléter et améliorer la cathédrale (même si le chapitre doit le remettre à l'ordre pour que les travaux avancent à un rythme régulier). C'est donc à la zone modifiée par ses soins dans son ensemble qu'il faut porter l'attention pour bien saisir l'importance de cette étape constructive et sa signification idéologique.

\section{Et si le portail occidental était... lausannois?}

L'approche typologique développée dans ce volume par Marcel Grandjean montre que les modèles potentiels du portail ou, du moins, les ensembles les plus similaires se situent en France, notamment en Indre-et-Loire, et s'inscrivent pour les plus importants d'entre eux (cathédrale de Tours, Vendôme, Thouars, etc.) entre les années 1430 et 1540. Il demeure toutefois difficile de relier ces exemples lointains avec Lausanne, où la reprise du tympan ouvert en remplage constitue un hapax, ou presque ${ }^{11}$. Si la carrière d'Aymon peut expliquer les liens avec la Loire notamment ${ }^{12}$, celle des maîtres d'œuvre est trop peu connue pour amener des éléments de réponse; originaires du Faucigny ou du

9. À l'est, il existe un chemin, mais il traverse le cimetière et donne sur la rue de la Cité-Derrière, moins importante.

IO. ... ut melius ipsa ecclesia decoretur dit le manuel du chapitre (ACV, Ac 13, p. 3, 25 septembre 1504, cité par E. Dupraz, La cathédrale de Lausanne, p. 486, n. 3).

II. On retrouve sa version miniaturisée à l'église de Saint-Saphorin en Lavaux (env. 1517-1521), due à Jean Contoz, l'un des deux maîtres d'œuvres du portail lausannois (M. Grandjean, "Les architectes "genevois” dans le Pays de Vaud à la fin de l'époque gothique (1470-1533)»).

I2. Voir à ce propos l'étude de M. Grandjean dans ce volume. 
Bas-Chablais, bourgeois de Genève, ils semblent actifs dans la région lémanique uniquement ${ }^{13}$. Ils pourraient bien sûr avoir exécuté un plan envoyé d'ailleurs, comme c'est déjà l'usage dans la région depuis longtemps ${ }^{14}$, mais la difficulté du chantier - l'adaptation d'un portail à une situation existante, unique et complexe - et le lien quasi organique existant entre l'ancien et le nouveau portail parlent plutôt en faveur d'une œuvre imaginée par Magyn et Contoz et leurs maîtres de l'ouvrage. Si l'hypothèse d'un modèle extérieur n'est pas à exclure, on peut tout de même imaginer un autre cas de figure: celui d'un modèle local, en l'occurrence situé dans la cathédrale même. Car à y regarder de plus près, le portail ajouté par Aymon n'est peut-être pas aussi moderne qu'il en a l'air.

\subsection{Un portail en imitation?}

Rappelons rapidement les principales caractéristiques du portail, telles que décrites par Marcel Grandjean dans ce volume: monumentalité du parti, embrasure profonde de l'arcature, absence de trumeau et tympan largement ajouré, à riche remplage (voir pl. XI). La première caractéristique tient beaucoup à la morphologie si particulière du porche du XIII siècle, dont l'arcade antérieure surhaussée, comme on l'a déjà souligné, forme une ouverture étroite et haute. Afin d'éclairer le vestibule désormais fermé, la solution employée - un portail à tympan ajouré est particulièrement habile. Elle démontre le souci des maîtres d'œuvre de jouer avec la structure préexistante de manière très ingénieuse; la profondeur de l'embrasure en découle également: elle répète la forme de niche de l'ancienne arcade d'entrée. Mais cela n'est pas tout: autrefois, les fidèles voyaient, à travers le premier porche ouvert, la seconde arcade, celle surmontée d'une ouverture fermée par la claustra de pierre, qui forme une sorte de tympan ajouré ${ }^{15}$, puis la troisième arcade, celle donnant dans le passage routier.

I3. M. Grandjean, L'architecture religieuse en Suisse romande et dans l'ancien diocèse de Genève à la fin de l'époque gothique, p. 211 sq.

I4. En 1386, la comtesse Bonne de Bourbon reçoit des plans de Paris pour la construction du château qu'elle souhaite édifier à Ripaille, près de Thonon (M. Grandjean, «Le château de Vufflens», p. 118).

I5. Les analyses archéologiques en cours mettent en doute l'authenticité de l'entier de ces éléments, qui semblent avoir été très retouchés; selon Peter Kurmann, les figures 
L'absence de trumeau, si remarquable et apparemment si problématique au moment du chantier puisqu'elle fait l'objet de discussion au sein du chapitre, est également un signe tangible de la reprise du type des "portails" préexistants: on n’a sans doute pas assez souligné l'originalité des trois arcades successives de l'église à ce propos. Sans trumeau, mais également sans linteau ni tympan, elles sont donc complètement libres, alors qu'elles auraient très bien pu montrer un dispositif plus riche, notamment afin de porter des figures dans les ébrasements et des scènes sur le tympan ${ }^{16}$. Ici, c'est l'architecture qui prime, les faisceaux de colonnettes se succédant en trois doubles rangées successives, accusant la profondeur de l'ensemble, au détriment très clair de la sculpture, cantonnée aux chapiteaux des colonnettes et à quelques éléments de modénature. Reste la question de la quatrième arcade, celle qui donnait du passage routier dans la nef, servant sans doute de porte à l'église. Sa forme et son type ne sont pas connus hélas, les fouilles du début du $\mathrm{XX}^{\mathrm{e}}$ siècle ne s'étant pas préoccupées de cette question; mais si l'on suit la logique impliquée par la succession des arcades sans trumeau du massif occidental et leur rétrécissement progressif, on peut supposer que la porte donnant dans la nef était aussi d'une forme très simple ${ }^{17}$. Nous rejoignons ici, mais par un autre biais, l'hypothèse émise par Marcel Grandjean en 1975 à propos du mur séparant le passage de la nef:

L'existence des colonnettes orientales des faces intérieures des piles asymétriques, qui ne reçoivent actuellement aucune retombée [...] offre [...] la possibilité d'envisager un mur percé d'une porte médiane, la "grande porte» citée en 1455, encadrée de deux arcades assez profondes reposant d'une part sur les colonnettes maintenant inutiles des piles asymétriques et de l'autre sur les colonnettes qui auraient flanqué des contreforts ou un épaississement du mur autour de la porte; on

de la Vierge, de la Reine de Saba et de Salomon auraient pu être ajoutés justement par Aymon, pour "enrichir» le portail (P. Kurmann [éd.], La cathédrale Notre-Dame de Lausanne, p. 130). Peut-être l'aspect actuel de cette partie de la cathédrale ne date-t-il donc que du début du XVI ${ }^{\mathrm{e}}$ siècle?

I6. Dans une configuration un peu différente, on peut penser au portail intérieur de l'église de la Madeleine à Vézelay, entre le narthex et la nef, à trumeau et tympan (env. 1135-1150); il est toutefois bien plus large qu'à Lausanne, ceci étant justement permis par le recours à une structure à trumeau.

I7. C'est aussi ainsi que l'ont représentée les concepteurs du plan publié en 1975 (M. Grandjean, «La cathédrale actuelle», p. 135). 
aurait ainsi une composition rappelant celle de la face interne du croisillon nord, dans sa partie inférieure ${ }^{18}$.

Car, en effet, la porte nord du transept, qui donnait autrefois dans la galerie orientale du cloître, ne comporte pas non plus de trumeau et son tympan en arc brisé est plein, mais non décoré de sculptures (il pouvait être peint en revanche). Enfin, si l'on se fie au plan d'Erasme Ritter (1763), la porte du jubé donnant accès au chœur liturgique était aussi à simple ouverture et se situait dans l'axe des quatre autres du massif occidental et du passage routier. On n'en connaît pas la forme, mais si l'on se réfère au jubé de l'église de Valère à Sion qui pourrait bien s'en inspirer ${ }^{19}$, on a aussi affaire à une porte en arc brisé, sans tympan, similaire à celles décrites plus haut dans le massif. Les jubés à portail dotés d'un trumeau existent aussi, mais ils sont très rares ${ }^{20}$.

Constatons, à ce stade, la sobriété, mais aussi l'uniformité des différentes arcades connues servant de portail à la cathédrale, qui ne jouent pour exprimer leur fonction que sur des formes purement architecturales (colonnettes, modénature). La seule exception, pour le XIII ${ }^{\mathrm{e}}$ siècle, c'est bien sûr le portail peint compris dans le porche rajouté en cours de chantier sans doute vers 1230 , sur le flanc sud de la nef, en remplacement sans doute d'une porte sans trumeau ${ }^{21}$. Ce remaniement cherche vraisemblablement à pallier la difficulté d'entrer dans l'église de ce côtélà, mais peut-être aussi le déficit d'un portail digne de ce nom à l'ouest. Alors que les autres portails font plutôt référence à des modèles anglonormands - comme une grande partie de l'architecture de l'édifice du $\mathrm{XIII}^{\mathrm{e}}$ siècle d'ailleurs ${ }^{22}$-, le nouveau portail, à trumeau, linteau et tympan figurés, est quant à lui beaucoup plus proche des exemples français contemporains. L'analyse des portes et portails de la cathédrale d'Amiens de Dieter Kimpel et Robert Suckale, établissant notamment une subtile et signifiante hiérarchie entre elles ${ }^{23}$, pourrait avoir tout son sens aussi à

I8. M. Grandjean, «Le magnum portale de la cathédrale de Lausanne et le passage routier de la "grande travée" ", p. 207 sq.

I9. E. Dupraz, La cathédrale de Lausanne, p. 516.

20. Cathédrale de Naumburg, 1249-1255.

2I. C'est du moins l'hypothèse de W. Stöckli et Ph. Jaton, "Chronologie du portail peint de la cathédrale de Lausanne».

22. M. Grandjean, «La cathédrale actuelle», p. 159-163.

23. D. Kimpel, R. Suckale, L'architecture gothique en France, 1130-1270, p. 11-17. 
Lausanne: la porte principale des fidèles est, certes, celle rajoutée ultérieurement à la construction sans doute pour résoudre des problèmes de circulation autour et dans l'église, mais c'est surtout la plus riche aux points de vue formel et iconographique. Si Alain Villes lit en la porteniche occidentale une porta coeli - on pourrait ajouter : une foederis arca -, peut-être ce message était-il insuffisant aux yeux du chapitre cultivé du XIII siècle. L'apport d'une iconographie mariale complexe, assez unique en son genre ${ }^{24}$, est peut-être la réponse, moins monumentale, mais plus orthodoxe dans ses formes et sa structure (à trumeau et tympan) impliquée par le modèle francilien et royal dominant, associée à une architecture dont l'esthétique est sans doute un peu dépassée aux yeux de chanoines dont certains ont étudié à Paris ${ }^{25}$ et connaissent sans doute les chantiers les plus modernes de leur temps.

Lorsque le nouveau portail est bâti au XVI ${ }^{\mathrm{e}}$ siècle, il répond d'ailleurs au même besoin de splendeur: les chanoines insistent auprès de l'évêque pour qu'il soit construit au-devant de l'ancien porche ut apud Deum et mundum habeat et consequatur majus meritum et res ipsa sit exellencior ${ }^{26}$. Mais peut-être la reprise de l'ancien dispositif à porte simple, sans trumeau, finalement opéré, doit-elle être lue comme un rappel historique, voire un signe de permanence.

\subsection{La tradition du lieu}

Cette question du souvenir, lié à la question cruciale de la memoria, semble trouver un écho particulier à l'époque d'Aymon de Montfalcon. Sous son épiscopat, un auteur, connu sous le seul prénom de Louis, rédige une chronique épiscopale qui relate l'histoire des évêques de Lausanne de Protais (640-env. 699) à Guillaume de Varax († 1466), fondée notamment sur une lecture critique du Cartulaire de Conon d'Estavayer et d'autres pièces d'archives auxquelles Aymon lui a donné accès ${ }^{27}$. Un élément concernant l'église cathédrale elle-même doit nous intéresser ici. La question de l'origine du siège de l'évêché, sis à

24. P. Kurmann, «Un cas exceptionnel».

25. J.-D. Morerod, «Le pays de Vaud et les universités aux XII ${ }^{\mathrm{e}}$ et XIII ${ }^{\mathrm{e}}$ siècles».

26. Archives cantonales vaudoises, Ac 13, p. 173 (cité par E. Dupraz, La cathédrale de Lausanne, p. 492, n. 3).

27. C. Santschi, Les évêques de Lausanne et leurs historiens des origines au XVIII siècle, p. $145-155$. 
Avenches, est notamment discutée par Louis; selon lui, la chapelle Saint-Symphorien n'est "manifestement pas assez splendide pour avoir pu être une église épiscopale. Au contraire, dit-il, il y avait à Lausanne, dès l'époque de Protais, une "magnifique église collégiale" " 28 . Il y avait bien une autre église cathédrale à Avenches, mais elle fut donc abandonnée en faveur de la collégiale de Lausanne promue dès lors au rang de cathédrale. L'ancienneté du siège lausannois de l'évêché est ici mise en évidence pour démontrer l'antériorité du chapitre par rapport au pouvoir épiscopal ${ }^{29}$. Mais la chronique insiste aussi, en fait, sur l'ancienneté de l'édifice, mettant en évidence la perdurance du pouvoir au travers de l'édifice qui le représente et qui fut "magnifique» dès ses origines.

Il ne faut pas sous-estimer la valeur à la cour d'Aymon d'un texte aussi rare dans le contexte régional de son temps ${ }^{30}$. Mais il permet aussi de procéder à une relecture de l'édifice gothique sous un angle mémoriel, celui de la tradition du lieu qui, sous différentes formes, se transmet au Moyen Âge notamment dans les cathédrales et les églises dévolues à un pèlerinage ${ }^{31}$. L'édifice actuel montre de nombreuses dispositions ou des éléments qui peuvent être lus comme des garants de l'identification de la permanence de l'église depuis ses origines; opérons un bref passage en revue. En plan, c'est la disposition singulière du premier vestibule à deux absides latérales qui peut être rapprochée d'un édifice antérieur, connu de manière fragmentaire par des fouilles archéologiques. Situé sans doute en avant de la façade principale de la cathédrale de l'an mil ${ }^{32}$, il présentait un plan vraisemblablement triconque ou quadrilobé. Sa fonction n'est pas connue, mais son plan peut rappeler un baptistère ou une fontaine, selon Louis Blondel ${ }^{33}$. L'emplacement actuel du vestibule à absides, grosso modo à l'emplacement de la structure à trois ou quatre absides, pourrait donc plaider en faveur d'une évocation de l'édifice plus ancien dont la fonction était sans doute importante et la forme se devait d'être évoquée, une fois l'édifice disparu, par son remplaçant.

28. Ibid., p. 152 sq.

29. Ibid., p. 153.

3o. Ibid., p. 143.

3I. H. Horn, Die Tradition des Ortes.

32. Un doute subsiste à ce propos, les fondations du XIII e siècle ayant grandement perturbé le sous-sol de cette partie de l'église (Ph. Jaton, «Les cathédrales antérieures à l'édifice gothique", p. 53).

33. L. Blondel, «Les édifices antérieurs à la cathédrale actuelle», p. 45 sq. 
Une autre disposition quelque peu étonnante pourrait être expliquée ainsi: la chapelle du chœur, également en forme d'abside, qui dépasse, esseulée, du déambulatoire du XII ${ }^{\mathrm{e}}$ siècle. On suppose qu'un chevet à cinq chapelles rayonnantes avait été prévu dans la première phase du chantier de l'église actuelle, au vu des vestiges archéologiques; selon Werner Stöckli, ce chœur aurait été construit jusqu'au niveau des chapiteaux puis arasé à l'exception de la chapelle centrale, intégrée dans le bâtiment gothique ${ }^{34}$. Or, si l'on observe le plan de l'église de l'an mil, dans la version de Louis Blondel reprise par W. Stöckli, on remarque qu'elle possède justement un déambulatoire, le premier loin à la ronde ${ }^{35}$, doté d'une vaste chapelle centrale absidiale qui pourrait avoir constitué le modèle du nouveau chevet du XII ${ }^{\mathrm{e}}$ siècle, à nouveau pour des raisons de tradition ${ }^{36}$. Un gisant d'évêque, habituellement identifié comme celui de Roger de Vico Pisano, semble s'y trouver depuis le Moyen Âge ${ }^{37}$. En élévation, cette chapelle à l'intérieur possède des caractéristiques stylistiques archaïsantes, comme les pilastres cannelés (en fait, cannelés et rudentés en alternance), le bandeau sculpté qui suit la forme des piliers et, surtout, les chapiteaux de la partie basse. Ceux-ci pourraient dater de l'époque d'Amédée de Lausanne (1145-1159) et auraient pu avoir été récupérés comme des " "reliques" architecturales encastrées dans la nouvelle cathédrale en souvenir de cet évêque qui aurait mis en route les travaux de construction ${ }^{38}$.

Pour en venir enfin à l'intervention d'Aymon, il n'est pas que «son" portail qui fasse référence à l'histoire architecturale de la cathédrale. Ainsi, les murs qu'il fait édifier vers 1502-1505 pour boucher l'ancien passage routier méritent une grande attention (fig. 2). En effet, s'ils sont ajourés au nord et au sud par de grandes baies à remplage flamboyant typiques de leur temps, surmontées à l'extérieur par une frise frappée de sa devise, la partie basse de ces murs montre dans la nef des

34. W. Stöckli, «Les édifices antérieurs à la cathédrale actuelle», p. 17.

35. Ibid., p. 25.

36. L'église de l'an mil conservait elle-même des vestiges de la crypte de l'église précédente (W. Stöckli, «Les édifices antérieurs à la cathédrale actuelle», p. 25 et 29). Plus récemment, Philippe Jaton a contesté ce plan en supposant un chevet à trois absides, mais sans guère de justifications (Ph. Jaton, "Les cathédrales antérieures à l'édifice gothique»).

37. C. Huguenin, G. Cassina, D. Lüthi (dir.), Destins de pierre, p. 144.

38. P. Kurmann (éd.), La cathédrale Notre-Dame de Lausanne, p. 52. 


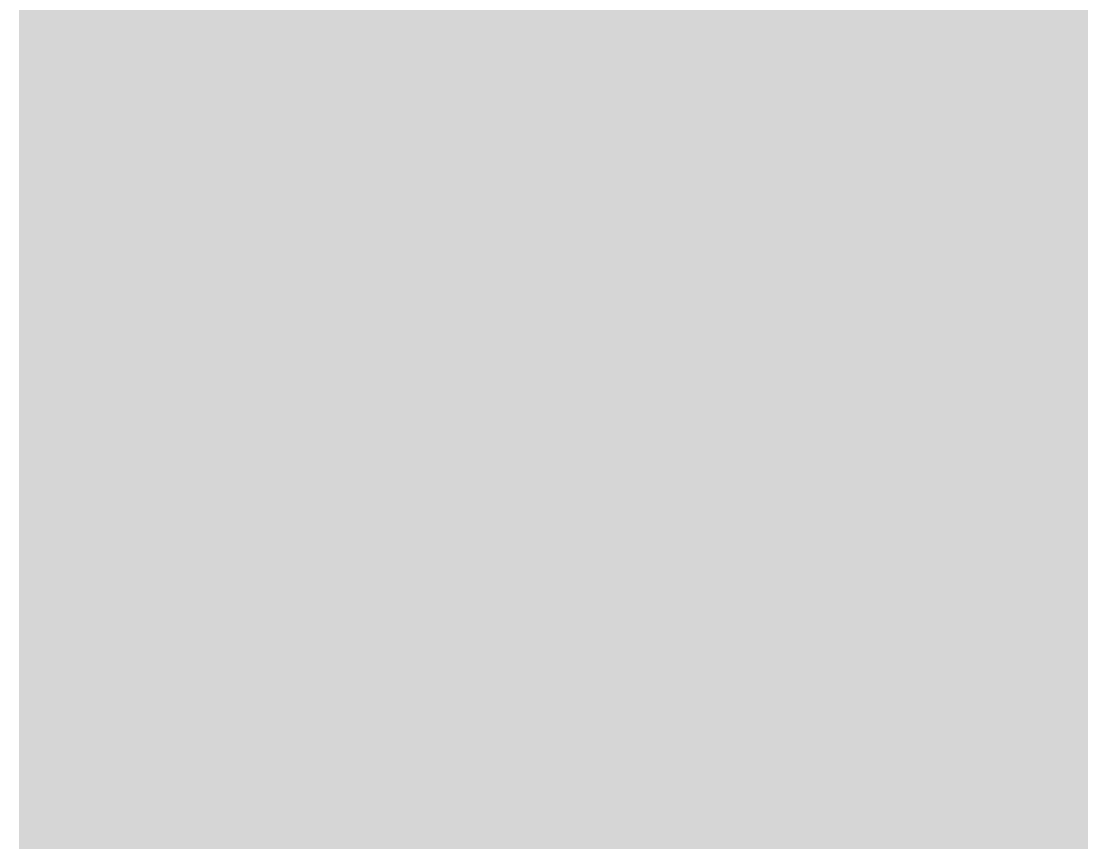

Fig. 2 - Grande travée de la cathédrale de Lausanne et sa fermeture nord. Photographie Jeremy Bierer, 2012.

arcatures trilobées dont le modèle est copié des travées voisines du début du XIII siècle. À quelques détails de modénature près (la profondeur est moins accusée dans les parties du XVI $\mathrm{XI}^{\mathrm{e}}$ siècle), la copie est parfaite, à tel point qu'il a fallu attendre les observations d'Hans Reinhardt en $1951^{39}$, confirmées par les travaux plus poussés de Marcel Grandjean en 1975, pour comprendre qu'elles dataient de l'époque d'Aymon alors même que ce dernier "signe» de ses armes de manière tout à fait exemplaire les travaux qu'il fait réaliser. La porte qui perce la paroi nord est particulièrement intéressante: si à l'intérieur, elle se fond dans le décor de style XIII siècle (porte rectangulaire sous un arc trilobé servant de tympan), à l'extérieur son encadrement à succession d'arcatures et de larmiers à modénature flamboyante (tore à listel, gorges, etc.) est quant à lui caractéristique, comme la baie qui le surmonte, de l'art de son temps. Il s'agit proprement d'une "véritable œuvre d'archéologue du

39. H. Reinhardt, "L'église-porche de la cathédrale de Lausanne». 
$\mathrm{XVI}^{\mathrm{e}}$ siècle» comme le souligne $\mathrm{H}$. Reinhardt ${ }^{40}$. Un dernier élément, aujourd'hui hélas supprimé, doit retenir l'attention: la chaire polygonale qui marquait le centre de la tribune raccourcie lors de la suppression du passage routier. Démontée lors de la construction du nouvel orgue en 2003, elle était l'un des éléments majeurs des apports de l'évêque dont elle portait aussi bien la devise que les armoiries. Datée 1505, elle signalait la fin des travaux de fermeture de la grande travée qui précèdent, on le sait, la construction du portail. Sa fonction nous échappe, comme celle d'ailleurs des tribunes hautes, faute de sources à ce propos. Toutefois, toujours dans l'hypothèse d'une tradition du lieu à laquelle Aymon aurait pu être sensible, on pourrait faire le lien entre les dispositions anciennes de la cathédrale du XIII ${ }^{\mathrm{e}}$ siècle à double tribune échelonnée avec des dispositifs similaires dans certaines églises ottoniennes et romanes à tribunes très profondes contenues dans le massif occiden$\mathrm{tal}^{41}$. Dans ces dernières, la fonction est souvent impériale ${ }^{42}$ : pourraiton voir dans la chaire lausannoise, dont la présence est si étonnante, le rappel d'une tribune plus ancienne où le roi des Romains Rodolphe - dont il faut rappeler qu'il est le souverain de la ville de Lausanne aurait pu assister à la dédicace de l'église en 1275 , célébrée par le pape Grégoire $\mathrm{X}$ ? Et, par ricochet, le souvenir des tribunes impériales à trône surélevé, marque de la dignité suprême? Selon Jean-Daniel Morerod, l'Église de Lausanne "tire toute sa légitimité des droits que lui ont accordés les rois rodolphiens et des empereurs, leurs successeurs, attachant de ce fait une grande importance à son immédiateté " ${ }^{43}$; il ne serait donc pas absurde de la part d'Aymon, qui vient justement de faire réécrire l'histoire de sa principauté par le mystérieux Louis et qui pouvait avoir connaissance de la cérémonie de 1275 puisque son déroulement était

40. Ibid., p. 355 ; repris par M. Grandjean, «Le magnum portale de la cathédrale de Lausanne et le passage routier de la "grande travée" ", p. 202.

4I. Les exemples «classiques" sont la chapelle palatine d'Aix-la-Chapelle (792-805), l'abbaye de Corvey (873-885), Saint-Philibert de Tournus (env. 1028-1056), l'abbaye de Gandersheim (XII siècle), etc. Synthèses récentes sur le sujet: Ch. Sapin (dir.), Avantnefs et espaces d'accueil dans l'église, entre le $I V^{e}$ et le XII e siècle; K. Krüger, Die romanischen Westbauten in Burgund und Cluny.

42. À partir du modèle de la chapelle palatine et du trône de l'empereur situé sur la tribune occidentale d'Aix-la-Chapelle (K. Corsepius, "Der Aachener "Karlsthron" zwischen Zeremoniell und Herrschermemoria»).

43. J.-D. Morerod, Genèse d'une principauté épiscopale, p. 496. 
décrit sur des affiches sans doute placardées lors de la fête de la dédicace à la fin du $\mathrm{XV}^{\mathrm{e}}$ siècle ${ }^{44}$, de faire acte de déférence à un souverain qui lui assure un prestige historique certain et a permis à son territoire de se constituer face aux velléités des Savoie ${ }^{45}$. L'hypothèse est sans aucun doute hardie, mais elle pourrait expliquer la forme si intrigante de la partie occidentale de la cathédrale.

\section{Une petite collégiale?}

Si la tradition est respectée par Aymon de Montfalcon et peut-être même ranimée par lui, il n'en est pas moins un homme de son temps, sensible aux formes modernes de dévotion, comme en témoignent plusieurs œuvres qu'il a commanditées ${ }^{46}$. Dans le contexte régional, le portail occidental apparaît ainsi de manière assez isolée comme une œuvre d'une qualité et d'une ambition sans commune mesure alors ${ }^{47}$, tellement exceptionnel qu'il faut peut-être se demander s'il ne revêt pas plusieurs significations. Nouvelle porte de la cathédrale rénovée, certes, mais estce son unique fonction?

À vrai dire, plusieurs indices donnent à penser qu'Aymon a vraisemblablement eu pour ambition la fondation d'une collégiale plutôt que d'une simple chapelle ${ }^{48}$. La richesse de la dotation et le luxe de l'aménagement ne sont pas les seuls éléments de ressemblance avec ces fondations typiques de la fin du Moyen Âge. Le souci mémoriel dont témoigne Aymon est plus généralement un élément moteur de la fondation des collégiales «à vocation funéraire [qui] reflète[nt] un aspect de la spiritualité du fondateur, mais aussi sa réaction devant la généralisation de l'enterrement ad sanctos, en instaurant un lieu d'inhumation placé sous la bienveillance d'un collège de chanoines $"{ }^{49}$. La situation de la

44. H. Meylan, «La consécration de la cathédrale, 20 octobre 1275 », p. 9 sq.

45. J.-D. Morerod, Genèse d'une principauté épiscopale, p. 293-295.

46. Voir en particulier dans ce volume l'article de B. Pradervand.

47. Le portail principal de la collégiale de Berne (1490-1500) est très original aussi dans sa conception et sa sculpture, mais il reste traditionnel dans sa structure architecturale. Sur les portails de cette époque, M. Grandjean, L'architecture religieuse en Suisse romande et dans l'ancien diocèse de Genève à la fin de l'époque gothique, p. 654-660.

48. Voir la contribution de K. Berclaz ici-même.

49. J. Noblet, En perpétuelle mémoire, p. 15 sq. 
Fig. 3 - Cathédrale Saint-Pierre à Genève et chapelle des Macchabées à droite. Gravure de Robert Gardelle (détail), vers 1730 (Bibliothèque de Genève).

chapelle éloignée du chœur de la cathédrale, éloignée aussi des tombeaux des autres évêques, contribue à l'isolement du prélat et de son tombeau. Les collégiales régionales de fondation privée sont rares: notons celle de Neuchâtel, servant de nécropole aux comtes éponymes, fondée au $\mathrm{XII}^{\mathrm{e}}$ siècle ${ }^{50}$, celle de Valangin (1507) pour les seigneurs du lieu ${ }^{51}$ et à Genève, celle du cardinal de Brogny à la cathédrale de Genève, dite chapelle des Macchabées (1406) ${ }^{52}$. Cette dernière, bien qu'a priori très différente de la chapelle d'Aymon puisqu'il s'agit d'un édifice à part entière construit au flanc de la cathédrale sous la forme d'une chapelle à trois travées et abside polygonale, est pourtant proche de Lausanne par certains aspects (fig. 3). Ainsi, son luxe extrême et, surtout, sa position à l'ouest de la cathédrale, latérale, ne sont pas sans rappeler la

50. Helvetia Sacra, II/2, p. 392-399.

5I. Helvetia Sacra, II/2, p. 536 sq.

52. M. Grandjean, «La chapelle des Macchabées à Genève (1397-1405), le maître d'œuvre Colin Thomas et les débuts de l'architecture gothique flamboyante». 
chapelle d'Aymon, éloignée aussi du chœur de la cathédrale. À Lausanne toutefois, au contraire de Genève où la collégiale du cardinal de Brogny dominait la façade de la cathédrale Saint-Pierre, singulièrement modeste quant à elle, la chapelle d'Aymon de Montfalcon ne peut guère «s'exprimer" à l'extérieur sinon par le biais des trois baies percées dans les épaisses parois de la tour nord inachevée et qui sont assez discrètes (mais néanmoins frappées des armes du prélat). Dès lors, ne faut-il pas voir dans le portail occidental la marque architecturale extérieure de la chapelle si elle avait été une collégiale?

Cette hypothèse relève essentiellement des constats des recherches typologiques de Marcel Grandjean dans ce volume, qui mettent en évidence un corpus à notre sens non négligeable de collégiales privées montrant un même «motif» de portail à tympan-verrière, la plupart sans trumeau. La plus ancienne est sans doute celle de Thouars (1505-1512, façade en 1513-1515) suivie peu après dans la région du Val de Loire par Les Roches Tranchelion (env. 1510-1527), Ussé (env. 1522-1538) et Montrésor (env. 1522-1541), ces deux dernières faisant un emploi plus ou moins développé du vocabulaire formel à l'antique ${ }^{53}$. Selon Julien Noblet, «[c]es quatre façades développent un parti novateur qui permet de revisiter les formules du XVe siècle et de les mettre au goût du jour par un riche décor sculpté de qualité. [...] les collégiales doivent par leur architecture refléter la magnificence des fondateurs et de leur famille» ${ }^{54}$. À Thouars, la façade juxtapose le portail, profond, richement orné, et une galerie qualifiée de loggia par J. Noblet qui y voit un apport ultramontain. À Lausanne, la galerie n'existe pas, mais elle trouve une forme de correspondance dans les niches qui abritent les statues des saints. Si la ressemblance formelle est frappante et si la chronologie s'accorde bien avec celle de Lausanne, il n'est toutefois pas possible de relier les deux édifices de manière plus tangible ${ }^{55}$. Retenons en tous les cas qu'ils emploient au même moment le même "motif" pour exprimer une fonction

53. Ussé mêle formes gothiques et renaissantes, Montrésor est d'architecture renaissante.

54. J. Noblet, En perpétuelle mémoire, p. 144.

55. Sur les liens entre Aymon de Montfalcon et le pays de Loire, nous renvoyons à l'article de M. Grandjean ici-même. 
similaire ${ }^{56}$, qui fait dire à Julien Noblet qu'il s'agit d'un des éléments de l'«architecture parlante» dont se parent alors les collégiales ${ }^{57}$ : faut-il donc penser qu'en appliquant un portail «expressif», Aymon de Montfalcon et ses maîtres d'œuvre cherchaient à faire passer un message à qui savait le lire?

Les deux approches ici envisagées, celles de la tradition du lieu et celle de l'expression d'une fonction, ne se contredisent pas, mais peuvent être considérées séparément. Elles mettent en évidence la complexité des questions que les interventions d'Aymon de Montfalcon soulèvent. La qualité des travaux et leur caractère exceptionnel dans le contexte régional autorisent une lecture qui dépasse les seules formes architecturales; à la lumière de la personnalité de l'évêque et de ce que l'on peut deviner de sa culture artistique, il ne faut sans doute pas considérer ses ouvres comme de simples pis-aller - une fermeture plus ou moins habile d'un porche exceptionnel et d'un passage routier qui ne l'est pas moins -, mais au contraire, des occasions de mettre en exécution des projets architecturaux ambitieux. On pourra regretter que le prélat n’ait pas eu un goût plus développé de la pierre, car les œuvres connues laissent entrevoir un raffinement qui n’a d'égal que dans les plus riches évêchés de son temps.

\author{
Dave LüTHI \\ Université de Lausanne
}

56. ... même si à Thouars, le chapitre doit louer la mémoire de Louis II de la Trémoille, ancien général des armées royales et non pas celle d'un prélat et même si à Lausanne, la collégiale sans doute rêvée par l'évêque est restée en projet...

57. C'est le titre de son chapitre consacré à l'architecture des édifices (J. Noblet, En perpétuelle mémoire, p. 101-159). 


\section{BIBLIOGRAPHIE}

BLONDEL, Louis, "Les édifices antérieurs à la cathédrale actuelle», in La Cathédrale de Lausanne, éd. par Eugène Bach, Louis Blondel, Adrien Bovy, Bâle, Birkhäuser, 1944, p. 27-59 (Les Monuments d'art et d'histoire du canton de Vaud, 2).

Cathédrale de Lausanne. $700^{e}$ anniversaire de la consécration solennelle, catalogue d'exposition, Lausanne, Musée historique de l'AncienÉvêché, 1975.

Corsepius, Katharina, "Der Aachener “Karlsthron” zwischen Zeremoniell und Herrschermemoria", in Investitur- und Krönungsrituale. Herrschaftseinsetzungen im kulturellen Vergleich, hrsg. von Marion Steinicke, Stefan Weinfurter, Köhl, Böhlau, 2005, p. 359-375.

Dupraz, Emmanuel, La cathédrale de Lausanne. Etude historique, Lausanne, Librairie Th. Sack, 1906.

GrandjEAn, Marcel, «La cathédrale actuelle. Sa construction, ses architectes, son architecture", in La cathédrale de Lausanne, éd. par Jean-Charles Biaudet et al., Berne, Société d'histoire de l'art en Suisse, 1975, p. 45-174.

—, "Le magnum portale de la cathédrale de Lausanne et le passage routier de la "grande travée" ", Revue suisse d'art et d'archéologie, 32/3 (1975), p. 193-220.

—, "Les architectes “genevois" dans le Pays de Vaud à la fin de l'époque gothique (1470-1533)", in Des archives à la mémoire: mélanges d'histoire politique, religieuse et sociale offerts à Louis Binz, Genève, Société d'histoire et d'archéologie de Genève, 1995, p. 159-216.

—, "Le château de Vufflens (vers 1415 - vers 1430). Notes sur sa construction, son esthétique et sa valeur défensive», Revue suisse d'art et d'archéologie, 2 (1995), p. 89-136.

—, "La chapelle des Macchabées à Genève (1397-1405), le maître d'œuvre Colin Thomas et les débuts de l'architecture gothique flamboyante», Genava, 52 (2004), p. 3-46. 
—, "CEuvres majeures de la ferronnerie en Suisse romande à la fin de l'époque gothique", in Petit précis patrimonial. 23 études d'histoire de l'art offertes à Gaëtan Cassina, éd. par Dave Lüthi, Nicolas Bock, Lausanne, Edimento, 2008, p. 199-216.

_, L'architecture religieuse en Suisse romande et dans l'ancien diocèse de Genève à la fin de l'époque gothique, Lausanne, Cahiers d'archéologie romande, 2015 (Cahiers d'archéologie romande, 157-158).

Helvetia Sacra, II/2. Die weltlichen Kollegiatstifte der deutsch-und französischsprachigen Schweiz, bearbeitet von mehreren Autoren, redigiert von Guy P. Marchal, Bern, Schwabe, 1977.

Horn, Hauke, Die Tradition des Ortes. Ein formbestimmendes Moment in der deutschen Sakralarchitektur des Mittelalters, München, Deutsche Kunstverlag, 2015.

Huguenin, Claire, Cassina, Gaëtan, Lüthi, Dave (dir.), Destins de pierre. Le patrimoine funéraire de la cathédrale de Lausanne, Lausanne, Cahiers d'archéologie romande, 2006 (Cahiers d'archéologie romande, 104).

Jaton, Philippe, «Les cathédrales antérieures à l'édifice gothique», in $L a$ cathédrale Notre-Dame de Lausanne. Monument européen, temple vaudois, éd. par Peter Kurmann, Lausanne, La Bibliothèque des arts, 2012, p. 51-53.

Kimpel, Dieter, Suckale, Robert, L'architecture gothique en France, 1130-1270, Paris, Flammarion, 1990 ( $1^{\mathrm{re}}$ éd. orig. allemande 1985).

KRÜGER, Kristina, Die romanischen Westbauten in Burgund und Cluny: Untersuchungen zur Funktion einer Bauform, Berlin, Mann, 2002.

Kurmann, Peter, «Un cas exceptionnel: le portail peint de Lausanne et son iconographie», Mémoire vive, pages d'histoire lausannoise, 15 (2006), p. 20-28.

- (éd.), La cathédrale Notre-Dame de Lausanne. Monument européen, temple vaudois, Lausanne, La Bibliothèque des arts, 2012.

Meylan, Henri, «La consécration de la cathédrale, 20 octobre 1275 ", Revue historique vaudoise, 83 (1975), p. 9-15.

Morerod, Jean-Daniel, «Le pays de Vaud et les universités aux XII e et XIII siècles", in Écoles et vie intellectuelle à Lausanne au Moyen Âge, éd. par A. Paravicini Bagliani, Lausanne, Université de Lausanne, 1987, p. 25-52. 
—, Genèse d'une principauté épiscopale. La politique des évêques de Lausanne (IXe-XIV siècles), Lausanne, Bibliothèque historique vaudoise, 2000 (Bibliothèque historique vaudoise, 116).

Noblet, Julien, En perpétuelle mémoire: collégiales castrales et sainteschapelles à vocation funéraire en France (1450-1560), Rennes, Presses universitaires de Rennes, 2009.

Pérouse de Montclos, Jean-Marie, Architecture: description et vocabulaire méthodiques, Paris, Éd. du patrimoine, 2011.

Reinhardt, Hans, "L'église-porche de la cathédrale de Lausanne", in Art du haut Moyen-Âge dans la région alpine, Olten/Lausanne, Graf, 1954, p. 355-357.

SantsChI, Catherine, Les évêques de Lausanne et leurs historiens des origines au XVIII siècle: érudition et société, Lausanne, Société d'histoire de la Suisse romande, 1975 (Mémoires et documents de la Société d'histoire de la Suisse romande, $3^{\mathrm{e}}$ série/XI).

SAPIN, Christian (dir.), Avant-nefs et espaces d'accueil dans l'église, entre le IV et le XII e siècle, Paris, Éd. du CTHS, 2002.

STÖCKLI, Werner, "Les édifices antérieurs à la cathédrale actuelle", in $\mathrm{La}$ cathédrale de Lausanne, éd. par Jean-Charles Biaudet et al., Berne, Société d'histoire de l'art en Suisse, 1975, p. 13-30.

Stöckli, Werner, Jaton, Philippe, "Chronologie du portail peint de la cathédrale de Lausanne», Mémoire vive, pages d'histoire lausannoise, 15 (2006), p. 10-19.

Utz, Sabine, "Dans la gueule du lion. Les appliques en bronze de Notre-Dame de Lausanne", in Déclinaisons gothiques. Le portail Montfalcon de la cathédrale de Lausanne, éd. par Claire Huguenin, catalogue d'exposition, Gollion, Infolio, 2017, p. 38-43.

Villes, Alain, "La cathédrale actuelle: sa chronologie et sa place dans l'architecture gothique", in La cathédrale Notre-Dame de Lausanne. Monument européen, temple vaudois, éd. par Peter Kurmann, Lausanne, La Bibliothèque des arts, 2012, p. 55-90. 